\title{
Mapeo nacional de capacidades en comunicación y salud pública en Argentina
}

\section{Palabras clave}

información

comunicación

encuesta

profesión

salud

\author{
Daniela Bruno \\ Valeria Zapezochny \\ Andrea Jait \\ Lucila Tufro \\ Carolina Casullo \\ (Buenos Aires, Argentina) \\ Ministerio de Salud de la Nación Argentina
}

Carolina Deguer

(Buenos Aires, Argentina)

Gobierno de la Ciudad de Buenos Aires

\begin{abstract}
Resumen
La Coordinación Nacional de Información Pública y Comunicación (CYPyC) y el Programa de Funciones Esenciales de Salud Pública (FESP), ambos dependientes del Ministerio de Salud de la Nación Argentina, desarrollaron a mediados de 2010 una encuesta nacional que tuvo como principal objetivo trazar una línea de base que contemple las capacidades institucionales en comunicación de los ministerios nacional y provinciales, con el fin de mejorar su gestión e institucionalizar las áreas de comunicación. La muestra estuvo conformada por 113 comunicadores integrantes de áreas, direcciones y programas del Ministerio de Salud de la Nación y de sus pares provinciales. Los principales ejes temáticos indagados fueron necesidades, demandas y expectativas de asistencia técnica y capacitación de los colegas comunicadores del sector, trayectoria profesional y formación específica, condiciones de trabajo, nivel de conocimiento de la oferta formativa específica en su provincia, caracterización del sistema de medios provincial/municipal y del sector asociativo, entre otros. En este artículo presentamos los principales hallazgos de la encuesta y algunas evaluaciones preliminares de la implementación en curso de iniciativas que con fines de empoderamiento se diseñaron a partir de las conclusiones que arrojó la encuesta nacional.
\end{abstract}




\title{
Mapping of Communication and Public Health Capabilities in Argentina
}

\begin{tabular}{c}
\hline Keywords \\
\hline information \\
communication \\
survey \\
profession \\
health
\end{tabular}

\begin{abstract}
In 2010 the Coordinación Nacional de Información Pública y Comunicación (CYPyC) and the Programa de Funciones Esenciales de Salud Pública (FESP), both of which depend on the Argentinean Health Ministry, developed a national survey of aimed towards laying down a baseline which would contemplate the institutional communication capabilities of the national and provincial ministries, so as to improve their management and institutionalize their communications. The sample was composed of 113 communicators who were part of areas, managements, and programs of the National Health Ministry and of provincial ministries. The main areas of study were the needs, demands and expectations for technical assistance and the communications professionals' capabilities, their experience and specific education, work conditions, knowledge of educational opportunities within their province, characteristics of the provincial/municipal media system and that of the associative sector, among others. In this article we present the survey's main findings and some preliminary evaluations of the current implementation initiatives which, for the purpose of empowerment, were drawn from the national survey's conclusions.
\end{abstract}

\section{Cómo citar el artículo}

Bruno, D. et al. (2011). Mapeo nacional de capacidades en comunicación y salud pública en Argentina. Revista de Comunicación y Salud, 1(2), pp. 5-18.

DOI: http://doi.org/10.35669/revistadecomunicacionysalud.2011.1(2).5-18

\section{Introducción}

La Coordinación Nacional de Información Pública y Comunicación del Ministerio de Salud de la Nación Argentina (CYPyC), con el respaldo técnico y financiero del Programa de Funciones Esenciales de Salud Pública (FESP) ${ }^{1}$, desarrolló a inicios del 2010 el primer Plan Estratégico de Información Pública y Comunicación (PEIPyC) de ese Ministerio. Uno de los objetivos del PEIPyC fue el "empoderamiento de los comunicadores para que estos se reconocieran y los

\footnotetext{
${ }^{1}$ El Proyecto FESP se desarrolla en el país desde 2007 con el apoyo del Banco Mundial y tiene el propósito de mejorar la salud de la población con una mirada integral dirigida a aumentar las capacidades de los sistemas de salud, la prevención y promoción de la salud, la participación comunitaria y la inclusión social.
} 
reconocieran (decisores y equipos técnicos) como profesionales capaces y legítimos para ocupar posiciones más estratégicas en la toma de decisiones de la política sanitaria". Con ese propósito, a mediados de 2010 se indagaron las principales necesidades, demandas y expectativas de asistencia técnica y capacitación de los colegas comunicadores de áreas y programas del Ministerio de Salud de la Nación y de las Provincias ${ }^{2}$. Esta experiencia inédita en el país permitió contar con un primer 'mapa' de capacidades, habilidades y condiciones de trabajo de nuestros colegas de todo el país.

El análisis de las más de un centenar de encuestas respondidas fue la base para la posterior realización de una serie de iniciativas a nivel nacional, regional y provincial que combinaron la articulación inter-programática, la capacitación continua, el intercambio de experiencias y la definición de una agenda común. En este artículo presentamos los principales hallazgos de la encuesta y algunas evaluaciones del proceso de empoderamiento en curso que se fundamentó en gran medida en aquel estudio.

Durante los encuentros presenciales con colegas de las provincias, en momentos posteriores a la encuesta, se advirtió la necesidad de construir y apoyar desde el nivel nacional iniciativas para posicionar a la comunicación como dimensión estratégica de las políticas sanitarias. Una y otra vez aparecía en los debates la demanda de acciones con vistas a fortalecerlos para que accedieran a recursos, desarrollaran capacidades, se reconocieran y los reconocieran (decisores y equipos técnicos) como profesionales capaces y legítimos para ocupar posiciones más estratégicas en la toma de decisiones de la política sanitaria.

La encuesta permitió verificar que un escollo fundamental para lograr ese requerimiento es la tradicional concepción de la comunicación (incluso por parte de algunos de nuestros colegas comunicadores) como el manejo de los medios y el cuidado de la imagen pública de la autoridad sanitaria. Por supuesto, sumando a ello la habitual referencia a ese don, arte u oficio para 'traducir' el saber biomédico en mensajes 'culturalmente adecuados' que colaboren en la prevención de enfermedades, la promoción de hábitos saludables o el compromiso de la población con el logro de metas previamente establecidas por los decisores de la política sanitaria. Ni en la práctica ni las expectativas de rol profesional se dimensiona la potencia de la comunicación para además transformar la cultura organizacional, crear y recrear otros sentidos posibles y mejorar las interacciones con interlocutores, públicos de interés y ciudadanos.

El diseño de la encuesta y de los posteriores encuentros partió, sin embargo, de una concepción diferente. Concebimos al campo de acción de la comunicación en la política pública y sanitaria en particular de manera más amplia, actuando por los menos en tres dimensiones

\footnotetext{
${ }^{2}$ El sistema sanitario argentino se divide en tres subsectores: el público, de la seguridad social y el privado, que abarca 24 sistemas de salud pública provinciales. El país cuenta con alrededor de 300 ofertas de coberturas de salud, entre obras sociales provinciales, empresas prepagas, seguros privados de salud, mutuales, y el Instituto Nacional de Servicios Sociales para jubilados y pensionados (PAMI). El subsector de la salud pública contempla las jurisdicciones provinciales y municipales, además de la nacional; la seguridad social implica una multiplicidad de instituciones agrupadas de acuerdo a la población a las que se dirigen; las obras sociales y privadas incluyen organizaciones y servicios también heterogéneos. Según el Plan Federal de Salud 2010/2016, el Estado Nacional es el garante final de la salud pública y por tanto debe promover el acceso de las personas a los servicios de salud, la calidad de estos y la equidad, que en el caso argentino se ha desarrollado sobre la base de la atención primaria de la salud, jerarquizando las acciones de prevención y promoción, el compromiso federal de cobertura, calidad y acceso, redes de servicio, población bajo responsabilidad nominada, acceso a los medicamentos y fortalecimiento de hospitales.
} 
interconectadas:

1. La dimensión de la política donde se negocian los contenidos, los recursos y el sentido general de cualquier iniciativa en materia de política sanitaria en la que los comunicadores podemos abogar para que las autoridades sanitarias y decisores de diferentes niveles y sectores conozcan iniciativas, las apoyen y contribuyan a su buen resultado, legitimándolas y convocando a otros para que participen en ella.

2. La dimensión organizativa donde los comunicadores podemos aportar a la construcción de espacios formativos, de encuentro y concertación, ya sea con otras áreas del mismo Ministerio o del Estado, o actores sociales estratégicos (formadores de opinión, universidades, gremios, sindicatos, organizaciones no gubernamentales y sociales o de base comunitaria, etc.) para activar redes de mediación y de concertación.

3. La dimensión sociocultural donde se ubican las acciones comunicativas que interpelan a la sociedad en general o a sus grupos específicos con el fin de comunicar la acción de gobierno, instalar y construir socialmente un tema o un nuevo enfoque sobre un tema, colaborar en la prevención de enfermedades y la promoción de la salud, y comprometer a actores sociales en una iniciativa de cambio ${ }^{3}$.

Así pensada, la comunicación adquiere otro alcance y significado, sus interlocutores o audiencias se multiplican y diversifican, y los objetivos de la estrategia, aunque contemplan objetivos informativos, son complementados por otros objetivos pedagógicos o de interacción (movilización).

Desde esta concepción ampliada consecuentemente se redefinen los desafíos de la formación y el empoderamiento de los comunicadores. Esta dimensión estratégica de la gestión de la política pública requiere ser desarrollada (como capacidad organizacional) por todo la organización (y no sólo por el Departamento de Comunicación) y le plantea a todos el desafío de una mirada comunicacional más allá de lo instrumental (el folleto, el afiche o la gacetilla), más sensible a la trama cultural en la que están inmersos los actores internos y externos, y más política (consciente de los contextos políticos e institucionales que pueden dar marco a los procesos de cambio).

En otras palabras, la comunicación no es solamente asunto de comunicadores, y comunicar no es solamente imaginar eslóganes ingeniosos, formatos novedosos o lenguajes sorprendentes. También es hacer política entendida como animación de redes y procesos organizativos que hagan posibles objetivos de cambio propuestos de manera concertada.

\footnotetext{
${ }^{3}$ Estas dimensiones son una adaptación de las propuestas por Jaramillo López Juan Camilo (2004). Modelo de Comunicación Pública Organizacional e Informativa para Entidades del Estado. Bogota: USAID/Casal \& Associates Inc.
} 


\section{De las cuestiones metodológicas}

Durante el primer semestre de 2010 el equipo técnico de la CIPyC con la asistencia técnica y financiera del FESP se abocó al diseño, el testeo previo, la puesta a punto del cuestionario y el lanzamiento del estudio. Se realizó una reunión de coordinación con los Responsables Operativos Regionales (ROR) del FESP para introducir ajustes al cuestionario y consensuar la logística más conveniente para la administración y recepción de la encuesta. Los ROR fueron los encargados de identificar a los comunicadores en el nivel provincial, garantizar que recibieran los cuestionarios vía electrónica y asegurar que las respuestas llegaran en tiempo y forma a la CIPyC. Para la administración, seguimiento y monitoreo de la encuesta se diseñó una mesa de consultas para atender dudas y sugerencias de las provincias.

A los efectos de este estudio se consideraron comunicadores aquellas personas que tuvieran a su cargo o estuvieran directamente involucradas en tareas que los equipos reconocieran como relativas a la comunicación social (diseño y realización de campañas, producción de materiales informativos y educativos, prensa, movilización social y comunitaria, etc.) independientemente de que pertenecieran a áreas, direcciones o programas de prensa o comunicación o similares -de hecho participaron un importante número de comunicadores de áreas de promoción o educación para la salud- o tuvieran formación técnica específica en la materia. No era necesario tener un título en comunicación o especialidad afín.

Durante los meses de mayo, junio y primera quincena de julio se realizó el envío y recepción de los cuestionarios. A fines de julio se inició la carga de datos para su posterior análisis que concluyó en el mes de agosto.

El principal objetivo de la encuesta fue contar con información actualizada y fehaciente sobre el grado de institucionalidad de las áreas de comunicación, qué sabían hacer (conocimientos y destrezas técnicas), qué deseaban hacer (expectativas y motivaciones) y qué efectivamente podían hacer (condiciones y oportunidades concretas que ofrece el ámbito de trabajo) nuestros colegas comunicadores.

La muestra estuvo conformada por 113 comunicadores integrantes de áreas, direcciones y programas del Ministerio de Salud de la Nación y de sus pares provinciales.

Los principales ejes temáticos indagados fueron trayectoria profesional y formación técnica específica, condiciones de trabajo, actividades habituales en su desempeño profesional actual, demandas de capacitación, conocimiento de la oferta formativa específica en su lugar de residencia, caracterización del sistema de medios provincial/municipal y del sector asociativo, caracterización del vínculo nación/provincias, entre otros. 


\section{Resultados}

\subsection{Distribución por niveles y áreas}

Del total de encuestas procesadas (113), el 67,25\% correspondió al nivel provincial mientras que el $32,74 \%$ al nivel nacional. Al menos una persona contestó el cuestionario en cada una de las provincias y en el conjunto de respuestas recibidas hubo un buen balance entre la visión del nivel nacional y provinciales. Sin embargo es importante reconocer que la cantidad de respuestas recibidas en el nivel nacional estuvo muy por debajo de las expectativas. Poco menos de la mitad de los comunicadores del nivel nacional contactados respondió la encuesta y casi todos los que respondieron integraban la CIPyC. Por el contrario en el nivel provincial, probablemente gracias a la tarea de los ROR y del seguimiento de la CIPyC, casi todos los contactados respondieron la encuesta.

Como ya señalamos anteriormente, podían responder la encuesta personas que tuvieran a su cargo o estuvieran directamente involucradas en tareas que se reconocieran como de comunicación social (diseño y realización de campañas, producción de materiales informativos y educativos, prensa, movilización social y comunitaria, etc.), independientemente de que pertenecieran a áreas, direcciones o programas de prensa y comunicación, o tuvieran formación técnica específica en la materia. Esto explica el hecho que el $40 \%$ de los encuestados integrara áreas de comunicación y/o prensa que dependen directamente de la autoridad sanitaria máxima; el $45,13 \%$ perteneciera a direcciones, programas o entes autárquicos (principalmente obras sociales y hospitales) y el $14,15 \%$ estuviera inserto en áreas de promoción de la salud o similares.

\subsection{Formalización de las áreas de prensa y comunicación}

Cerca del $90 \%$ de las áreas de comunicación y prensa cuentan con algún tipo de reconocimiento formal en la estructura ministerial: por Resolución Ministerial 15,21\%, por Disposición Ministerial el $4,43 \%$ y por decretos o documentos de menor jerarquía un $69,56 \%$. En la mayoría de los casos este reconocimiento formal es reciente. En promedio no supera los 4 años.

\subsection{Formación técnica superior}

La mitad de los encuestados cuenta con título terciario o universitario en comunicación. Hay un importante porcentaje de comunicadores que no cuenta con ningún título de grado $(26,54 \%)$. El porcentaje de comunicadores que ha realizado estudios de postgrado es apenas del 5,3\%. Existe un porcentaje importante de personas que trabajan en comunicación pero que han estudiado otras disciplinas $(16,81 \%)$. En general se trata de diseñadores gráficos y profesionales de otras ciencias sociales o de la salud.

Fueron excepcionales aquellos casos en los que los consultados manifestaron tener conocimiento de algún tipo de oferta formativa en comunicación a nivel terciario y/o universitario en sus provincias. 


\subsection{Trayectoria profesional y principales actividades de la profesión}

La mitad de los consultados tiene al menos 7 años de trayectoria profesional y sólo un $9 \%$ tiene menos de cuatro. Más de la mitad de los comunicadores tiene menos de cuatro años en su cargo actual. Esto coincide con la tendencia reciente de formalización de las áreas de prensa y comunicación mencionada anteriormente, lo que a su vez podría estar indicando el paulatino reconocimiento de la función de información pública y comunicación en salud pública. No se debería descartar que la antigüedad en el cargo, sobre todo en áreas políticamente sensibles, como las áreas de prensa y comunicación (que dependen directamente de los ministros), se deba a los ciclos electorales.

Al ser consultados sobre a qué tipo de tareas han dedicado mayor tiempo en su trayectoria profesional los comunicadores señalan la realización de campañas de comunicación y la elaboración de materiales de información y sensibilización $(27,27 \%)$, actividades de prensa $(26,31 \%)$ y tareas asociadas a la producción de materiales educativos y la coordinación de espacios (talleres, reuniones, charlas, etc.) con esos fines educativos en el nivel comunitario $(22,48 \%)$.

Cuando los interrogamos sobre su desempeño actual los comunicadores encuestados respondieron que no desempeñan una sola tarea. Entre las que les demandan más tiempo y esfuerzo aparecen las siguientes en orden de importancia:

1. Campañas $27,24 \%$ (básicamente la producción de mensajes radiales, televisivos y gráficos).

2. Capacitación $21,59 \%$ (diseño de cartillas, coordinación de talleres en la comunidad, etc.).

3. Prensa 18,60\% (elaboración de gacetillas y comunicados, cobertura de eventos y producción de información con fines periodísticos).

4. Diseño gráfico y de contenidos web $16,61 \%$.

\subsection{Demandas de capacitación}

Los comunicadores que participaron en la encuesta demandan capacitación en las siguientes categorías elaboradas a partir de las respuestas recibidas:

1. Planificación y evaluación de estrategias comunicacionales $(17,55 \%)$. Bajo este título se agruparon demandas relativas al diseño y gestión de estrategias de comunicación o bien al diseño, monitoreo y evaluación de proyectos sociales y de comunicación, etc.

2. Uso de TICs y desarrollo de contenidos web (12,9\%).

3. Comunicación y salud $(11,11 \%)$. Agrupamos en esta categoría a demandas en torno a la aplicación de abordajes teórico-prácticos de la comunicación en temas o enfoques relevantes para las políticas sanitarias muy diversos como VIH/sida, ITS, enfermedades no transmisibles, enfoque de derechos, concepciones de desarrollo, etc. Un número importante de respuestas agrupadas en esta categoría simplemente utilizó la denominación 
genérica 'comunicación y salud’ sin mayores precisiones.

4. Comunicación en situaciones de crisis/emergencias/riesgos (10,39\%).

5. Producción de materiales de educativos y de comunicación (9,31\%).

Si discriminamos las demandas de capacitación en función del tipo de área a la que pertenecen, los comunicadores que trabajan en áreas de prensa/comunicación demandan capacitación en comunicación de crisis y emergencias $(17,21 \%)$ planificación y evaluación de estrategias comunicacionales $(17,2 \%)$ y desarrollo de campañas de sensibilización e información $(12,29 \%)$.

En el caso de los comunicadores que trabajan en áreas de educación/promoción de la salud las demandas son en planificación y evaluación de estrategias comunicacionales $(27,76 \%)$, comunicación y salud $(19,44)$ y producción de materiales de comunicación $(13,88 \%)$.

En el caso de los comunicadores que trabajan en otros programas (no son de prensa y comunicación, ni de promoción de la salud) demandan capacitación en manejo de TICs y diseño de contenidos web $(15,2 \%)$, producción de materiales de comunicación $(12 \%)$, planificación y evaluación de estrategias comunicacionales (12\%) y comunicación popular/comunitaria (12\%).

\subsection{Mejora del desempeño}

Cuando los encuestados fueron consultados sobre qué podría mejorar su desempeño, las intervenciones que se propusieron espontáneamente en orden de importancia fueron:

Tabla1. Propuestas mejora del desempeño

\begin{tabular}{|l|r|}
\hline PROPUESTAS & \multicolumn{1}{c|}{$\%$} \\
\hline Capacitación de recursos humanos & $19,15 \%$ \\
\hline Trabajo en equipo y relación con otras áreas y organizaciones & $14,55 \%$ \\
\hline Mayor presupuesto para el área & $14,55 \%$ \\
\hline Infraestructuras/equipamiento & $14,17 \%$ \\
\hline Formalización del área & $11,87 \%$ \\
\hline Más recursos humanos & $7,66 \%$ \\
\hline Mejores salarios & $5,36 \%$ \\
\hline Redes de comunicadores & $3,44 \%$ \\
\hline Divulgación de resultados & $1,14 \%$ \\
\hline Otros & $8,04 \%$ \\
\hline
\end{tabular}

Fuente: elaboración propia

Si sólo se toman en cuenta las respuestas del nivel nacional, estos son los resultados: 
Tabla 2. Propuestas mejoras del desempeño (nacional)

\begin{tabular}{|l|r|}
\hline PROPUESTAS & \multicolumn{1}{|c|}{$\%$} \\
\hline Capacitación de recursos humanos & $20 \%$ \\
\hline Trabajo en equipo y relación con otras áreas y organizaciones & $20 \%$ \\
\hline Infraestructuras/equipamiento & $14 \%$ \\
\hline Redes de comunicadores & $12 \%$ \\
\hline Más recursos humanos & $10 \%$ \\
\hline Divulgación de resultados & $4 \%$ \\
\hline Otros & $10 \%$ \\
\hline
\end{tabular}

Fuente: elaboración propia

\subsection{Expectativas de rol profesional. Conocimientos, habilidades y aptitudes fundamen- tales en el desempeño profesional}

Respecto de las principales habilidades que debería tener un comunicador para su desempeño profesional las más altamente valoradas tuvieron que ver con la escucha, la observación y comprensión del público destinatario, su contexto, su cultura y su situación, seguidas del manejo experto de lenguajes para la producción de mensajes escritos y audiovisuales.

En lo que respecta a los conocimientos se verifica cierta coherencia con la respuesta anterior ya que los más valorados refieren a las condiciones económicas, políticas, sociales y culturales de los ámbitos de intervención. En segundo lugar se mencionan conocimientos en temáticas específicas del campo de la salud (VIH, salud reproductiva, etc.). En tercer lugar, conocimientos relativos a los medios de comunicación y en cuarto la metodología de planificación.

En relación con las actitudes fundamentales para el desempeño profesional, la predisposición a la escucha y el diálogo, y el respeto a la participación y los valores democráticos fueron las más valoradas.

\subsection{Articulación con el nivel nacional}

El $67 \%$ de los comunicadores que trabajan en el nivel provincial tuvo alguna experiencia de articulación con el nivel nacional. De estos, el $75 \%$ califica la experiencia como excelente o muy buena. Al ser consultados sobre la función que debería cumplir el Ministerio de Salud de la Nación en relación con las provincias en materia de información pública y comunicación, las personas encuestadas consideran funciones prioritarias del nivel nacional la asistencia técnica y financiera, la creación y animación de redes de información y trabajo, y facilitar el acuerdo de lineamientos políticos y técnicos de acción. 


\section{Discusión}

Aunque resulta positivo el altísimo reconocimiento formal en la estructura institucional es importante tener en cuenta que se trata de un fenómeno reciente (en promedio no supera los 4 años) que, de acuerdo a las intervenciones que las personas encuestadas proponen para mejorar su desempeño, se da en un contexto de insuficiente capacitación, presupuesto, equipamiento tecnológico y RRHH. Resulta evidente que, aunque las áreas de prensa y comunicación mantienen una relación de dependencia directa con los ministros, esta cercanía con la máxima autoridad sanitaria no implica necesariamente el reconocimiento de la comunicación como una dimensión estratégica de la política sanitaria.

Esto nos lleva de manera ineludible a la discusión sobre la concepción de comunicación y las expectativas que sobre los comunicadores depositan otros trabajadores o profesionales del campo de la salud e incluso algunos colegas. Aunque es cierto que desde hace décadas se viene dando una revisión del sesgo biomédico, individual y psicológico hegemónico en el campo desde mediados del siglo pasado, lo que llevó a prestar una mayor atención a los contextos sociales y culturales específicos, persisten visiones lineales que enfatizan en la idea de pocos emisores calificados y muchos receptores. Los primeros controlan, editan, diseñan y deciden los contenidos de la información que los comunicadores traducen en mensajes y campañas para persuadir a los segundos de adoptar ciertos comportamientos y prácticas, desconociendo muchas veces singularidades y diferencias socioculturales. Como plantea Charles Briggs (2005) caracterizando al enfoque dominante de la comunicación asociado al modelo médico hegemónico:

"Las personas [...] suelen ser caracterizadas en los folletos de educación para la salud, particularmente en las escenas introductorias de éstos, como productores de un discurso y un comportamiento que encarnan la ignorancia y/o la resistencia [...]. La trama pasa a la introducción del conocimiento biomédico bien a través de la entrada en escena del profesional de la salud o por la presentación descorporeizada de información autorizada sobre la enfermedad que incluye terminología médica. Para el final de la narración los personajes no profesionales han incorporado esta información en sus voces y en sus conductas" (Briggs, 2005: 105 y 106).

El mismo Briggs advierte cómo este enfoque es muchas veces refrendado en la construcción noticiosa de los temas de salud:

"Los artículos periodísticos y los noticiarios televisivos comienzan también con las voces cargadas de autoridad de los profesionales de salud y prosiguen después con las de personas no profesionales que tratan de asimilar el conocimiento biomédico, que están en riesgo de sufrir una enfermedad particular, que expresan miedo y/o demuestran su ignorancia o resistencia" (Briggs, 2005: 106).

Partiendo del dato acerca de lo que efectivamente hacen los comunicadores (campañas en medios de comunicación masiva, producción de materiales educativos y coordinación de talleres, prensa institucional y diseño de contenidos web), y si tenemos en cuenta que fueron escasas las 
menciones al trabajo articulado con organizaciones de la sociedad civil, universidades, gremios, etc., es evidente la persistencia del modelo "pocos emisores calificados y muchos receptores". A pesar de la revisión crítica desde la Academia, seguimos trabajando como editores y reeditores de la voz calificada en salud.

En tanto y en cuanto la tarea de los comunicadores siga asociada (y en alguna medida subordinada) a la traducción del conocimiento biomédico, difícilmente se la reconocerá como aspecto decisivo de los procesos de organización, participación y cambio social que implican intercambios, acuerdos, negociaciones y construcción colectiva del significado de la experiencia humana.

La resolución de problemas de salud requiere de un intercambio de doble vía que permita conocer la opinión, las expectativas y las propuestas del otro y manifestar las propias en un proceso que entrecruza las diferencias culturales, sociales, políticas, estéticas y éticas de los individuos y los grupos. La comunicación es estratégica porque, si se construyen espacios de comunicación y se posibilita el desarrollo de las competencias dialógicas de las personas y grupos, se generan más posibilidades de cogestión y autogestión para mejorar la salud. Cuando las personas consultadas mencionaron las actividades más habituales en el desempeño profesional fueron excepcionales las menciones a la articulación intersectorial, la abogacía e incidencia en decisores y formadores de opinión y agenda, y la mediación pedagógica para la mejora de la calidad de los servicios de salud.

La pregunta que queda pendiente es cómo, en un contexto de reconocimiento formal pero a la vez de insuficiente capacitación, presupuesto, equipamiento tecnológico y RRHH en comunicación, los comunicadores nos constituimos en profesionales capaces y legítimos para ocupar posiciones más estratégicas en la toma de decisiones de la política sanitaria. ¿Debemos esperar a que nos empoderen para poner en acto este enfoque o intentamos comunicar con dichos y hechos esta otra concepción de la comunicación como animación de procesos y redes que transformen la cultura organizacional, creen y recreen sentidos y mejoren la interlocución con la ciudadanía? ¿Cuán oportunos y estratégicos pueden ser hoy nuestros aportes?

La búsqueda de mayor coordinación interprogramática, articulación intersectorial y trabajo en red de pares que figuran entre las intervenciones propuestas para mejorar el trabajo de los comunicadores y visibilización de resultados nos sugiere que la tarea de los comunicadores es muchas veces solitaria, aislada y poco reconocida. Pero sin desconocer lo anterior cabe preguntarse si esto que los comunicadores expresan no es en todo caso el emergente de una organización que aún padece una fragmentación y una desarticulación históricas. La comunicación es para nosotras una capacidad organizacional y no sólo una competencia técnica especifica. ¿No se trata de competencias básicas transversales a todos los equipos?

Afortunadamente las demandas de capacitación indicarían la intención de ampliar el campo de intervención antes que profundizar los saberes técnicos vinculados con las tareas que ya desarrollan cotidianamente. Asimismo las habilidades, conocimientos y aptitudes profesionales más (la escucha, la observación y comprensión del público destinatario, su contexto, su cultura y su situación; el conocimiento de las condiciones económicas, políticas, sociales y culturales 
de los ámbitos de intervención; etc.) privilegian la dimensión relacional antes que la instrumental de la comunicación. Sin embargo los encuestados no conocen -salvo excepciones- oferta formativa específica ${ }^{4}$. La oferta de estudios de posgrado específicos en comunicación y salud es muy escasa, geográficamente concentrada y con modalidad presencial.

Finalmente, la articulación con el nivel nacional fue valorada positivamente, adjudicándole funciones de rectoría tales como asistencia técnica y financiera; creación y animación de redes de información y trabajo; y acuerdo de lineamientos políticos y técnicos de acción. Las enumeradas coinciden en gran medida con la definición que desde el nivel nacional se hace de la rectoría.

A modo de cierre y proyección, el análisis de la información obtenida a través del estudio constituyó la base para la implementación de una serie de propuestas en correspondencia con el objetivo de empoderamiento de los comunicadores comprendido en el Plan Estratégico de la Coordinación Nacional de Información Pública y Comunicación.

Se elaboró un protocolo de funciones básicas de información pública y comunicación en salud pública que surgieron del análisis que se hizo de las tareas que efectivamente desarrollan los comunicadores de todo el país, pero también de aquellas prácticas emergentes presentes en experiencias innovadoras, además de las prospecciones elaboradas con el apoyo de especialistas con quienes se intercambiaron ideas sobre tendencias en el campo de la comunicación y la salud en rondas de consulta.

Este material -en instancia de validación- aspira a constituirse en una referencia válida para planear la estructura de un área o departamento de comunicación a nivel ministerial, elaborar los planes estratégicos y operativos anuales de información pública y comunicación, definir las competencias básicas y las condiciones labores mínimas con las que debe contar un equipo de comunicación, orientar en la selección de personal sobre la base de estas competencias, capacitar a los trabajadores de las áreas de prensa y comunicación o afines sobre la base de las competencias fundamentales que requiere la tarea, y orientar en la definición de los Términos de Referencia (TDR) de eventuales contratos, convenios y/o acuerdos de asistencia técnica con universidades, empresas y/o consultoras para apoyar y complementar la labor de los equipos de trabajo.

Con miras a posicionar estratégicamente información relativa a la representación social (incluyendo la mediática) de los problemas sanitarios y divulgar los resultados del monitoreo y evaluación de las intervenciones en comunicación y salud en la toma de decisiones de política sanitaria, la CIPyC y la Dirección de Epidemiología del Ministerio de Salud de la Nación iniciaron una serie de acciones conjuntas para promover la incorporación de nuestros colegas en las salas de situación jurisdiccionales a partir de un trabajo de comunicación integral, vinculado con la realidad socioeconómica, demográfica y epidemiológica de la población, las representaciones sociales y mediáticas de las principales problemáticas sanitarias y el derecho a la

\footnotetext{
${ }^{4}$ En el punto "resultados" no se hizo referencia a este ítem para priorizar otros ejes y respetar la extensión máxima permitida según las normas de publicación de la revista.
} 
salud de la población.

Con posterioridad a la realización de la encuesta se desarrollaron una serie de encuentros que reunieron a gran parte de los comunicadores consultados en una experiencia que combinó la articulación inter-programática, la capacitación continua, el intercambio de experiencias y la definición de una agenda común. En lo que respecta específicamente a la capacitación, a la vez que se intentó dar respuesta a algunas demandas ligadas al actual desempeño prioritario de las áreas de prensa y comunicación tales como comunicación de riesgos o comunicación en situaciones de emergencia o crisis, esto se complementó con encuentros de formación en planificación estratégica, abordajes teórico-prácticos más relevantes de la comunicación en el campo de la salud, promoción de la salud, entre otras. Una de las particularidades de estos encuentros fue la invitación y creciente participación de profesionales no comunicadores y autoridades a cargo de direcciones o programas a nivel nacional o provincial e incluso ministros.

En definitiva, el conocimiento exhaustivo de las necesidades, demandas y expectativas de asistencia técnica y capacitación de nuestros colegas comunicadores, no sólo aporta a la transformación del rol y del consiguiente reconocimiento profesional en una dimensión tan esencial de la gestión sanitaria como la comunicacional, sino también otorga contenido genuino a la función de rectoría que la Coordinación de Información Pública y Comunicación, en tanto instancia nacional, debe garantizar para que el trabajo cotidiano en las diversas áreas y programas contribuya sustantivamente a los objetivos de política sanitaria vigentes en Argentina.

\section{Referencias}

Aprea, Gustavo y Cabello, Roxana. (2004). Los procesos comunicativos en los proyectos de Desarrollo Humano. Un enfoque teórico-metodológico. En Aprea, Gustavo (comp.). Problemas de comunicación y desarrollo. Buenos Aires: UNGS-Prometeo.

Briggs, Charles. (2005). Perspectivas críticas de comunicación y hegemonía comunicativa: aperturas progresistas, enlaces letales. Revista Antropología Social. № 14, pp. 101-124. Disponible en http://redalyc.uaemex.mx/pdf/838/83801404.pdf. Recuperado el 29 de marzo de 2011.

Carvalho, Sergio. (2008). Promoción de la salud, empowerment y educación: una reflexión critica como contribución a la reforma sanitaria. Salud Colectiva. Vol. 4, n 3, pp. 334-348. Disponible en http://redalyc.uaemex.mx/pdf/731/73140306.pdf. Recuperado el 15 de abril de 2011.

Cimadevilla, Gustavo. (2004). Tocarle la cola al león. Una lectura del desarrollo a través de sus condiciones de intervención. En Aprea, Gustavo (comp.). Problemas de comunicación y desarrollo. Buenos Aires: UNGS-Prometeo.

Cortés, Carlos Eduardo. (1997). La comunicación al ritmo del péndulo. Medio siglo en busca del desarrollo. Mimeo. Disponible en http://www.catedras.fsoc.uba.ar/uranga/pendulo carlos_cortes.pdf. Recupe- 
rado el 11 de julio de 2011.

Jaramillo López, Juan. (2004). Modelo de Comunicación Pública Organizacional e Informativa para Entidades del Estado. Bogotá: USAID/Casal \& Associates Inc.

Organización Panamericana de la Salud/Fundación Kellogg. (2001). Manual de comunicación social para programas de promoción de la salud de los adolescentes. Washington D.C.: Organización Panamericana de la Salud. Disponible en: http://www.paho.org/Spanish/HPP/HPF/ADOL/comSocial.pdf. Recuperado el 3 de mayo de 2010.

Rey, Germán. (2002). Cultura y desarrollo humano: unas relaciones que se trasladan. Pensar Iberoamérica, Revista de Cultura. № 0. Disponible en: http://www.oei.es/pensariberoamerica/ric00a04.htm. Recuperado el 6 de mayo de 2011. 